\title{
Una alternativa complementaria a la formación: las píldoras
}

\author{
An alternative to additional training: pills
}

\author{
Nuria Rebollo Quintela, Eva María Espiñeira Bellón, \\ *Departamento de Filosofía y Métodos de Investigación en Educación, Universidade da Coruña, España
}

\begin{abstract}
Resumen
Este estudio analiza las necesidades formativas de 64 estudiantes de la Facultad de Ciencias de la Educación (Universidad de A Coruña) en la elaboración de su Trabajo de Fin de Grado (TFG) o Máster (TFM). Se aplicó un instrumento compuesto por 15 ítems a valorar en una escala Likert con cinco opciones de respuesta. Los resultados muestran que el alumnado presenta necesidades formativas relacionadas con el desarrollo del proceso de investigación y metodologías de cómo afrontarlos, con la preparación de su presentación y con las competencias relacionas con su exposición pública. Palabras clave: Educación Superior, evaluación de necesidades formativas, investigación educativa, formación
\end{abstract}

\begin{abstract}
This study analyzes the training needs of 64 students of the Faculty of Educational Sciences (University of A Coruña) in preparing its Final Degree (TFG) or Master's Degree (TFM). An instrument composed of 15 items to evaluate on a Likert scale with five response options was applied. The results show that students presents training needs related to the development of the research process and methodology of how to deal with the preparation of their presentation and the skills relate to his public exposure.
\end{abstract}

Keywords Higher education, needs assessment, educational research, training

La puesta en marcha del Espacio Europeo de Educación Superior (EEES) ha desencadenado una serie de cambios. Cada Estado miembro ha llevado a cabo reformas educativas para el diseño e implantación de los nuevos títulos de grado y máster. En España la normativa que regula estos estudios se rige por el Real Decreto 1393/2007, de 29 de Octubre, por el que se establece la ordenación de las enseñanzas universitarias oficiales y por el Real Decreto 43/2015, de 2 de febrero, por el que se modifica el citado Real Decreto. En ella, se establece que los estudios de grado y máster deben finalizar con la elaboración y defensa de un Trabajo de Fin de Grado (TFG) y Trabajo Fin de Máster (TFM), en el que se deben plasmar las competencias adquiridas por los/las estudiantes. Sin embargo la experiencia pone en cuestión que el alumnado tenga la suficiente formación para poder llevar a cabo este cometido de manera autónoma.

Una nueva forma de ofertar formación específica a necesidades inmediatas las encontramos en lo que se denomina “píldoras formativas”. Desde el área MIDE de la Universidad de A Coruña se han promovido acciones de este tipo entendidas como experiencias de innovación educativa orientadas a cubrir determinadas lagunas conceptuales, procedimentales y actitudinales.

\section{Las píldoras como innovación formativa}

El Espacio Europeo de Educación Superior ha dibujado un nuevo escenario de enseñanzas, que ha motivado no sólo un cambio a nivel estructural sino curricular y organizativo (Mateo, 2000), lo que ha supuesto un cambio de paradigma basado en el aprendizaje del estudiante por competencias (Rekalde Rodríguez, 2011), que conlleva, tal y como se establece en el Real Decreto 1393/2007 una apuesta por nuevos métodos para adquirir y evaluar esas competencias. Este cambio lleva parejo el uso de nuevas estrategias y metodologías de enseñanza centrados en el uso de las Tecnologías de la Información y la Comunicación (TICs). La era digital, nos permite ampliar los escenarios de aprendizaje y no restringirlos a un tiempo y un espacio, jugando un papel destacado ya que dan "respuesta a las demandas del aprendizaje continuo" (Sánchez González, 2012,p.860). Una manera de desarrollar este tipo de aprendizaje, lo encontramos en lo que se han venido denominando como píldoras de conocimiento, píldoras formativas, dosis de conocimiento,... (Fidalgo Blanco et al., 2012; López, 2005; Maceiras, Cancela y Goyanes, 2010; Martínez-Fernández, García-Beltrán, Pastor-Moreno y Blanco-Galán, 2009) como "intento de dar respuesta a necesidades de inmediatez en el acceso a la formación/información” (Sánchez Allende, 2008, p.37). Estas "píldoras” de conocimiento, tal y como destacan Aranguena, Ruíz e Urrutia (2010) son una nueva forma de impartir acciones formativas optando por un formato presencial con sesiones cortas (no más de 120 minutos de duración) con una orientación práctica, que se acompaña de una documentación en forma de guía rápida a la que el alumnado pueda recurrir cuando lo necesite.

Desde el área MIDE de la Facultad de Ciencias de la Educación de la Universidad de A Coruña y en colaboración con el grupo GIACE (Grupo de Investigación e Avaliación Educativa) se ha desarrollado una experiencia de innovación formativa diseñada en 
base a las necesidades observadas en el alumnado tutorizado en los años posteriores a la implantación de los nuevos títulos, unido a que la elaboración y defensa del TFG y del TFM es uno de los principales temores de los/las estudiantes, ya que consideran que no tienen la suficiente formación para afrontarlo, siendo muchas de las dudas que presentan comunes a todos/as ellos/as. Las píldoras se pueden convertir en una herramienta que permite esa formación inmediata y de economía de tiempo para los/as tutores/as, al tratarse de una formación grupal.

La experiencia ha partido de 9 puntos clave identificados por profesores/as tutores/as en el desarrollo de este tipo de trabajo y de las manifestaciones de los estudiantes en las tutorías. Para lo cual se impartieron 9 sesiones formativas que responden a estos puntos clave: 1) El TFG/TFM y el proceso de investigación, 2) Abordar una investigación cualitativa, 3) Organiza los datos de tu cuestionario, 4) Analiza tus datos con estadística básica, 5) Representa tus datos mediante gráficos/tablas, 6) Aprende a almacenar, recuperar y compartir recursos, 7) Mejora tu estilo de redacción y aprende a citar, 8) Cómo hacer la presentación del TFG/TFM, 9) Tono y voz en la presentación de tu trabajo.

Estas píldoras fueron ofertadas a todo el alumnado de la Facultad de Ciencias de la Educación de la Universidad de A Coruña matriculados en el TFG o TFM, dándole la oportunidad de cursar aquellas en las que identificaban una carencia formativa. El aforo estaba limitado a 80 asistentes, excepto en las píldoras 3,4 y 5 que se impartían en dos grupos reducidos de 25 personas, al ser necesario tanto el uso de ordenadores como programas estadísticos.

Todas ellas se daban en formato presencial con sesiones de 75 minutos de presentación y 30 minutos destinados a la resolución de dudas particulares aplicables a la realización de sus propios TFG o TFM. Se acompañaban de un material didáctico que se ponía a disposición del alumnado (http://giace.udc.es/files/pilulas-formativas-ISBN.pdf).

\section{Método}

Se emplea el método hipotético-deductivo, a través de una metodología cuantitativa con el fin de dar respuesta al siguiente interrogante ¿el alumnado tiene la suficiente formación para poder elaborar y defender el TFG o TFM? Por lo que el principal objetivo que se pretende alcanzar es determinar las necesidades formativas del alumnado que está cursando los estudios de grado o master.

\section{Participantes}

Si bien el número de participantes en las píldoras, es desigual, oscilando entre 36 y 78, la muestra productora de datos está conformada por 64 estudiantes, correspondiendo el 90.3\% a Grado (46.77\% Educación Infantil, 32.26\% Educación Primaria, 9.67\% Educación Social y 1.61 Logopedia) y 9.7\% a Master Universitario (4.84\% Dirección, Gestión e Innovación de Instituciones
Escolares y Socioeducativas, 1.61\% Psicopedagogía, y 3.23\% Master en Profesorado de ESO y Bachillerato, FP y Enseñanza de Idiomas) (figura 1).

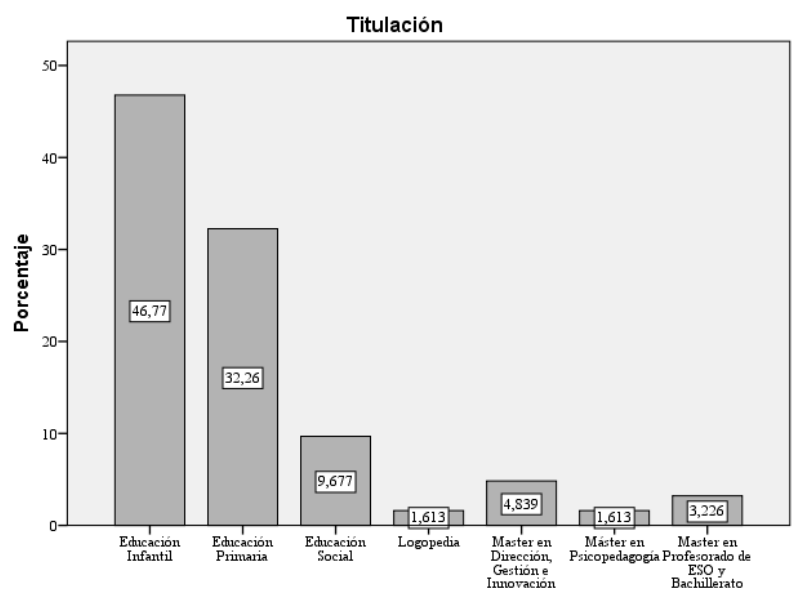

Figura 1. Distribución del alumnado por titulación

Nos encontramos con una muestra feminizada, $100 \%$ de mujeres, aspecto llamativo ya que la acción formativa estaba abierta a todo el alumnado del centro, que si bien es mayoritariamente femenino cuenta con alumnos entre sus discentes.

La selección muestral fue mediante muestreo no probabilístico de carácter accidental ya que se aplicó a todas las alumnas que asistieron y accedieron a cubrir el cuestionario de detección de necesidades.

\section{Instrumentos}

Se ha diseñado un cuestionario "ad hoc", compuesto por 15 ítems que se valoran en una escala de respuesta tipo Likert con 5 niveles siendo 1 “Nada” y 5 "Mucho” y una pregunta abierta referida a la principal causa que les ha motivado la realización de la acción formativa.

Para su elaboración se hizo a una primera redacción de 12 ítems sometidos a validación por jueces expertos del área MIDE, tutores/as de TFG/TFM, que recomendaron la incorporación de 3 ítems nuevos. Finalmente se procedió a la redacción del formato final incluyendo los ítems sugeridos.

La consistencia interna del instrumento diseñado se examinó mediante el análisis de fiabilidad. Los resultados muestran una alta fiabilidad (Maroco y García-Marques, 2006) ya que el alpha de Cronbach es de .89 .

\section{Procedimiento}

Para proceder a la recogida de la información se aplicó el cuestionario de detección de necesidades con anterioridad al desarrollo de las actividades.

\section{Resultados}

El tratamiento de los datos para su posterior análisis, se realizó con el paquete estadístico IBM Statistics Program for Social Sciencies (SPSS) en su versión 20. Los análisis planteados son descriptivos (n, media y desviación típica) de cada uno de los ítems que integran el cuestionario. 
Tabla 1.

Estadísticos descriptivos de las necesidades formativas

\begin{tabular}{|c|c|c|c|}
\hline \multicolumn{4}{|c|}{ Estadísticos descriptivos } \\
\hline & $\mathrm{n}$ & $\overline{\mathrm{x}}$ & SD \\
\hline $\begin{array}{l}\text { Identifico con claridad las partes del } \\
\text { TFG/TFM }\end{array}$ & 64 & 3,47 & ,872 \\
\hline $\begin{array}{l}\text { Soy capaz de estructurar mi TFG/TFM } \\
\text { de acuerdo con las fases del proceso de } \\
\text { investigación }\end{array}$ & 64 & 2,92 & ,965 \\
\hline $\begin{array}{l}\text { Me considero capaz de estructurar una } \\
\text { investigación cualitativa }\end{array}$ & 64 & 2,37 & ,845 \\
\hline $\begin{array}{l}\text { Identifico las principales técnicas de } \\
\text { investigación cualitativas }\end{array}$ & 63 & 2,56 & ,963 \\
\hline $\begin{array}{l}\text { Tengo conocimientos generales de } \\
\text { cómo abordar los datos en una } \\
\text { investigación cualitativa }\end{array}$ & 64 & 2,41 & ,886 \\
\hline $\begin{array}{l}\text { Tengo conocimientos generales de } \\
\text { cómo organizar los datos en una } \\
\text { investigación cuantitativa para su } \\
\text { tratamiento }\end{array}$ & 63 & 2,67 & ,950 \\
\hline $\begin{array}{l}\text { Soy capaz de analizar mis datos para } \\
\text { su análisis con estadística básica e } \\
\text { interpretarlos }\end{array}$ & 63 & 2,60 & ,908 \\
\hline $\begin{array}{l}\text { Soy capaz de representar los datos } \\
\text { mediante gráficos /tablas y } \\
\text { sintetizarlos }\end{array}$ & 63 & 2,70 & ,854 \\
\hline $\begin{array}{l}\text { Soy capaz de analizar mis datos para } \\
\text { su análisis con estadística básica e } \\
\text { interpretarlos }\end{array}$ & 63 & 2,79 & 986, \\
\hline $\begin{array}{l}\text { Se almacenar, recuperar y compartir } \\
\text { los recursos mediante herramientas } \\
\text { webs }\end{array}$ & 64 & 3,22 & 951 \\
\hline $\begin{array}{l}\text { Soy capaz de construir el marco } \\
\text { teórico de mi trabajo apoyándome en } \\
\text { fuentes documentales }\end{array}$ & 64 & 3,75 & 909 \\
\hline $\begin{array}{l}\text { Conozco la manera de citar los } \\
\text { trabajos utilizando la normativa APA }\end{array}$ & 64 & 3,62 & 951 \\
\hline $\begin{array}{l}\text { Tengo claro cómo elaborar la } \\
\text { presentación del TFG/TFM con la } \\
\text { ayuda de una herramienta informática }\end{array}$ & 64 & 3,16 & 963 \\
\hline $\begin{array}{l}\text { Conozco cual es la información más } \\
\text { importante para presentar en la } \\
\text { defensa del TFG/TFM }\end{array}$ & 64 & 2,77 & ,988 \\
\hline $\begin{array}{l}\text { Sé cuál es la mejor manera de usar el } \\
\text { tono y la voz para la presentación de } \\
\text { mi trabajo }\end{array}$ & 64 & 2,83 & 1,092 \\
\hline
\end{tabular}

Como podemos apreciar en la tabla 1 , nos encontramos con respuestas bastantes homogéneas ya que la desviación típica que presentan los ítems del cuestionario, sólo es superior a 1 en uno de ellos "Sé cuál es la mejor manera de usar el tono y la voz para la presentación de mi trabajo".

Las puntuaciones medias de los ítems comprenden $\overline{\mathbf{x}}=2.37$ del ítem "Tengo conocimientos generales de cómo abordar los datos en una investigación cualitativa" y $\overline{\mathbf{x}}=3.75$ de "Soy capaz de construir el marco teórico de mi trabajo apoyándome en fuentes documentales". Por lo que extraemos que la muestra encuestada, cree tener necesidades formativas en casi todos los aspectos referidos, obteniendo las puntuaciones más bajas los ítems referidos a la investigación cualitativa "Tengo conocimientos generales de cómo abordar los datos en una investigación cualitativa” ( $\overline{\mathbf{x}}=2.41)$ e "Identifico las principales técnicas de investigación cualitativas" $(\overline{\mathbf{x}}=2.56)$, así mismo, los ítems referidos a la investigación cuantitativa "Soy capaz de analizar mis datos para su análisis con estadística básica e interpretarlos", "Soy capaz de representar los datos mediante gráficos /tablas y sintetizarlos" y "Soy capaz de analizar mis datos para su análisis con estadística básica e interpretarlos” también obtienen medias inferiores a 3.

Si tenemos en cuenta las frecuencias de respuesta de los valores de la escala, siendo 1 nada, 2 poco, 3 algo, 4 bastante y 5 mucho, nos encontramos que en todos los ítems evaluados el 50\% de las respuestas se acumula en las categorías de poco y algo.

Por otro lado, del análisis de la pregunta abierta ¿cuál es la principal causa que te ha motivado a realizar esta actividad? se extrae que, la razón más aludida fue la necesidad de enfrentarse correctamente al TFG/TFM (enunciado por el $87 \%$ de las alumnas). Las causas restantes, a saber: "falta de formación en análisis de datos en investigación”, “falta de conocimientos estadísticos y desconocimiento del empleo del SPSS", "falta de formación sobre estudios de caso”, estaban relacionadas, concretamente con las píldoras formativas ofertadas - 3) Organiza los datos de tu cuestionario, 4) Analiza tus datos con estadística básica, 5) Representa tus datos mediante gráficos/tablas.

\section{Discusión}

La implantación de los títulos de grado y máster en la Enseñanza Superior española ha comportado innumerables cambios de tipo estructural, organizativo y curricular, pero ¿se han diseñado los nuevos títulos de forma que permitan al discente plasmar las competencias aprendidas en un trabajo final (de grado o máster), tal y como se establece en el RD 1393/2007 y en el RD $43 / 2015$ ?

A la vista de los resultados obtenidos, podemos decir que la muestra de alumnas encuestadas presenta necesidades formativas principalmente respecto a metodologías de investigación y procesamiento de la información, tanto cuantitativa como cualitativa. Estas pueden estar motivadas, en parte, por la carencia de materias propias de Métodos de Investigación en los estudios de Grados de Educación Infantil y Educación Primaria que se imparten en la Facultad de Ciencias de la Educación de la Universidad de A Coruña.

Así mismo, presentan necesidades formativas en aspectos relacionados con la preparación de la presentación del trabajo y con las competencias relacionas con su exposición pública, analizado a través de los ítems "Conozco cual es la información más importante para presentar en la defensa del TFG/TFM" y "Sé cuál es la mejor manera de usar el tono y la voz para la presentación de mi trabajo”. Estos resultados son 
destacables ya que las alumnas a lo largo de sus estudios de grado han empleado un gran abanico de metodologías en las que se ponían en práctica competencias comunicativas, como, discusión dirigida, mesa redonda, simulación, presentación oral,.. así como competencias relacionadas con la capacidad de análisis y síntesis trabajadas a través de metodologías como los trabajos tutelados, la recensión bibliográfica, esquemas, análisis de fuentes documentales,...

\section{Referencias}

Aranguena, E., Ruíz, P. e Urrutia, I. (2010). Retos y respuestas de la gestión de la información en la Corporación Tecnológica Tecnalia. Recuperado de http://eprints.rclis.org/15891/

Fidalgo Blanco, A., Sein-Echaluce Lacleta, M.L., Lerís López, D., Castañeda Toledo, O., Florentín Dueñas, P. y Sánchez Sarasa, M.P. (2012). Adaptabilidad versus eficacia en procesos de autoaprendizaje online: Proyecto Teaching Innova. Actas del XIV Simposio Internacional de Informática (pp. 13-18). Recuperado de

http://www.researchgate.net/publication/269410007_ Adaptabilidad_versus_eficacia_en_procesos_de_auto aprendizaje_online_Proyecto_Teaching-Innova

López, F. (2005). Metodología participativa en la enseñanza Universitaria. Madrid: Narcea.

Maceiras, R., Cancela, A. y Goyanes, V. (2010). Aplicación de nuevas tecnologías en la docencia universitaria. Formación Universitaria,3(1), 21-26. http://dx.doi.org/10.4067/S0718-50062010000100004

Maroco, J. y Garcia-Marques, T. (2006). Qual a fiabilidade do alfa de Cronbach? Questoes antigas e solucoes modernas? Laboratório de Psicologia, 4(1), 65-90. Recuperado http://publicacoes.ispa.pt/index.php/lp/article/viewFil e/763/706
Martínez-Fernández,R.,García-Beltrán,A.,Pastor-Moren o, S. y Blanco-Galán, L. (2009). ¿ ¿Se puede aprender una nueva forma de crear y usar contenidos educativos en la universidad? Revista de Educación a Distancia, $9 . \quad$ Recuperado de http://www.um.es/ead/red/M9/universidad.pdf

Mateo, I. (2000). La evaluación educativa, su práctica y otras metáforas. Barcelona: ICE-Horsori.

Real Decreto 1393/2007, de 29 de Octubre, por el que se establece la ordenación de las enseñanzas universitarias oficiales.

Real Decreto 43/2015, de 2 de febrero, por el que se modifica el citado Real Decreto Real Decreto 1393/2007, de 29 de octubre, por el que se establece la ordenación de las enseñanzas universitarias oficiales, y el Real Decreto 99/2011, de 28 de enero, por el que se regulan las enseñanzas oficiales de doctorado.

Rekalde Rodríguez, I. (2011). ¿Cómo afrontar el trabajo de fin de grado? Un problema o una oportunidad para culminar con el desarrollo de las competencias. Revista Complutense de Educación, 22(2), 179-193. http://dx.doi.org/10.5209/rev_RCED.2011.v 22.n2.38488

Sánchez Allende, J. (2008). Tómate una píldora... de conocimiento. Bit, 169, 37-39. Recuperado de http://www.researchgate.net/publication/28223154 _Tmate_un_apldora..._de_conocimiento

Sánchez González, M. (2012). El acceso abierto como fórmula hacia una Universidad más adaptada al contexto de cultura digital: tendencias y experiencias en el caso español [Número especial]. Estudios sobre el mensaje periodístico, 859-868. http://dx.doi.org/10.5209/rev_ESMP.2012.v 18.40964 